\title{
Duelo en discapacidad intelectual: los avances de una década
}

\author{
Bereavement in intellectual disability: the \\ advances of a decade
}

\section{Resumen}

El aumento de la esperanza de vida de la población con discapacidad intelectual (DI) ha puesto de manifiesto la falta de conocimiento acerca del duelo en este colectivo. Por esta razón, la presente revisión trata de conocer los avances de la última década respecto a las características del proceso en personas en duelo con DI, las herramientas de evaluación con las que se cuenta y los modelos de intervención propuestos. Para ello, se realizó una búsqueda de los estudios llevados a cabo en los años comprendidos entre 2007 y 2017 en diferentes bases de datos. Los resultados arrojan peculiaridades en el proceso, escasez de instrumentos de evaluación específicos para esta población y una falta de consenso en el tipo de intervención a llevar a cabo. Se discute acerca de la importancia de la sistematización de los estudios, el empleo de herramientas que permitan conocer las necesidades de apoyo de la persona con garantías psicométricas y la necesidad de intervenciones basadas en evidencias.

\section{Palabras clave}

Discapacidad intelectual, duelo, características, evaluación, intervención.

\section{Abstract}

The increased life expectancy in population with intellectual disability (ID) has revealed a lack of knowledge about grief in this group. For this reason, the present review tries to identify the advances made in the last decade in relation to the characteristics of the process of bereavement in people with ID, the available assessment tools and the main intervention models proposed in the field. To this end, a search for studies published from 2007 to $20 \mathrm{I} 7$ was carried out in different databases. The results show peculiarities in the process, scarcity of specific assessment tools for this population and a lack of consensus on the type of intervention to be delivered. A discussion is raised about the importance of systematization in studies, use of tools that allow knowing people's support needs with psychometric guarantees and the necessity of evidence-based practices.

\section{Keywords}

Intellectual disability, bereavement, characteristics, assessment, intervention.

\section{Leticia Cristóbal Fernández \\ <UO224099@uniovi.es> \\ Universidad de Oviedo}

\section{$M^{a}$ Ángeles Alcedo \\ Rodríguez \\ <malcedo@uniovi.es>}

Universidad de Oviedo

Laura E. Gómez Sánchez

<gomezlaura@uniovi.es>

Universidad de Oviedo

\section{Para citar:}

Cristóbal, L. et al. (2017): "Duelo en discapacidad intelectual: los avances de una década". Revista Española de Discapacidad, 5 (2): 53-72.

Doi: <https://doi.org/IO. 5569/23405 I04.05.02.03>

Fecha de recepción: 2I-03-20I7 Fecha de aceptación: 28-08-20I7 


\section{Introducción}

De acuerdo con las últimas estimaciones realizadas por la Organización Mundial de la Salud (OMS), casi un I $5 \%$ de la población mundial tiene alguna discapacidad (OMS, 20II). Esta cifra aumenta de forma progresiva debido a dos factores principales. Por un lado, el paulatino envejecimiento de la población -las personas mayores tienen un mayor riesgo de discapacidad-y, por otro, el incremento de enfermedades crónicas de salud asociadas a la discapacidad, como la diabetes o enfermedades cardiovasculares, que asolan principalmente a los países con bajos y medios ingresos (Hernández y Millán, 2015; OMS, 20I I). Al mismo tiempo, en las últimas décadas ha evolucionado la concepción de la discapacidad para abandonar el modelo médico, basado en los déficits de las personas, hasta el actual modelo social, que adopta un enfoque contextual entendiendo la discapacidad como resultado de la interacción entre la persona y el ambiente en el que se desenvuelve. De este modo, no son solo las deficiencias físicas, sensoriales o intelectuales las variables implicadas, sino que su funcionamiento depende en gran medida de las barreras y los facilitadores con los que se encuentra en su entorno. Cuando esta interacción da lugar a un desequilibrio que tiene un impacto en su participación plena y efectiva en la sociedad se produce la discapacidad (Cisternas, 2015; OMS, 200I).

En el territorio nacional, el último intento de cifrar el número de personas con discapacidad fue el realizado por el Instituto Nacional de Estadística (2008) en la Encuesta de Discapacidad, Autonomía personal y situaciones de Dependencia (EDAD). Según los datos de esta encuesta, el número de personas con discapacidad alcanza los 3.85 millones, lo que supone un $8.5 \%$ de la población. De este porcentaje, se estima que aproximadamente un I \% tiene discapacidad intelectual (DI). La definición propuesta por la Asociación Americana sobre Discapacidades Intelectuales y del Desarrollo (AAIDD), en su última edición, recoge que la DI se caracteriza por limitaciones significativas en el funcionamiento intelectual y en conducta adaptativa (i. e., habilidades conceptuales, sociales y prácticas), teniendo su origen antes de los I 8 años (Schalock et al., 2010). Para estas personas, los importantes avances médicos y las mejoras en sus condiciones de vida acaecidos durante las últimas décadas han propiciado un significativo aumento de su expectativa de vida, llegando a edades avanzadas y enfrentándose al proceso de envejecimiento y a los acontecimientos vitales que aparecen en esta etapa del ciclo vital. Entre las posibles crisis vitales de este período destaca la pérdida de personas significativas, que influye tanto en padres como en hijos (Millán, 2002) y que provocan importantes cambios en sus necesidades de apoyo (Aguado y Alcedo, 2004). Cuanto más tiempo viva una persona con DI, más probable es que experimente, al menos una vez en su vida, la muerte de alguien importante (Blackman, 2013) pues, aunque hasta no hace mucho los padres solían sobrevivir a sus hijos con DI, en el momento actual son los hijos los que están sobreviviendo a sus padres (Clute, 20Io). Se estima que aproximadamente el $54 \%$ de las personas con DI sufre la muerte de sus padres, en torno al $40 \%$ vive con padres de edad avanzada y el ro $\%$ convive con uno de los progenitores cuya edad supera los setenta años (Rodríguez Herrero et al., 201 3a). Esta situación novedosa en la vida de las personas con DI ha despertado un incipiente pero progresivo interés por su estudio en la literatura científica pues el afrontamiento de la pérdida, que supone uno de los momentos más complicados en la vida de cualquier ser humano, conlleva la experimentación de respuestas afectivas y emocionales intensas que acarrean retos decisivos.

Siguiendo la definición propuesta por Tizón (2004: I9), podríamos conceptualizar el duelo como el "conjunto de procesos psicológicos y psicosociales que siguen a la pérdida de una persona con la que el sujeto en duelo, el deudo, estaba psicológicamente vinculado". Se trata, por tanto, de una reacción emocional y comportamental causada por la ruptura de un vínculo afectivo que se manifiesta en forma de sufrimiento. Es un proceso dinámico y multidimensional, acompañado de signos y 
síntomas de índole diversa tales como sensación de ahogo, hipersomnia o insomnio, falta de ilusión, agotamiento y síntomas somáticos (Barreto et al., 2012; Díaz et al., 20I4; Meza et al., 2008). Cabe destacar que el afrontamiento del duelo no es un proceso psicopatológico en sí mismo, ya que la muerte es un proceso universal que forma parte de la vida y que suele conllevar una situación emocional de tristeza, lo cual no implica estar deprimido (Echeburúa y Herrán, 2007). A pesar de ello, cada ser humano experimenta la pérdida de una forma diferente, existiendo incluso variaciones en una misma persona. Dicha variabilidad está mediada por la interacción de factores personales y sociales (Neimeyer, 2002), especialmente por aspectos culturales del entorno en el que se desarrolla el individuo (Ruiz y Sainz, 2008). Las respuestas emocionales ante el duelo en la población general suelen ser de tristeza y ansiedad, a las que en muchas ocasiones se suma el temor a la soledad o sentimientos de culpa o alivio tras enfermedades de larga duración (Echeburúa y Herrán, 2007). Esta sintomatología habitualmente tiene una duración aproximada de seis meses a un año, que es el tiempo que se estima "normal" para la superación de los diferentes estadios del proceso: estupor o shock, confusión, búsqueda, aceptación y reintegración. Nótese que, aunque estas fases sean comunes en la mayor parte de las teorías tradicionales del duelo (Neimeyer, 2002; Tizón, 2004), no es en absoluto necesario atravesar cada una de ellas ni hacerlo en ese orden para superarlo (Pereira, 2010).

A pesar de que no es fácil establecer diferencias entre un curso normal del proceso y uno patológico -pues nos encontramos ante un continuo de signos y síntomas que pueden ser normales en función de las personas, culturas o épocas (Ruiz y Sainz, 2008) - la literatura científica se ha afanado tradicionalmente en clasificar los tipos de duelo de diversas formas, destacando entre ellas la distinción de duelo anticipatorio, crónico y retardado. El primero de ellos, el duelo anticipatorio, hace referencia a la expresión del dolor antes de una muerte anunciada. Puede ser experimentado por los familiares del enfermo o por el propio moribundo, quien sufre por la separación de sus seres queridos, la pérdida de su cuerpo o la incertidumbre, entre otros posibles motivos (Bermejo, 2005; García, 20I 2). Por su parte, el duelo crónico se refiere a la imposibilidad de la persona a retomar el estilo de vida previo al fallecimiento. Tiene una duración excesiva (en comparación con el período que se considere normal en su cultura) y cursa con intrusión constante de recuerdos, fantasías con el pasado y falta de conexión con el momento presente. En cambio, se habla de duelo retardado cuando no manifiestan una reacción emocional, inhibiendo los sentimientos propios que se derivan del fallecimiento de un ser querido (Muñoz et al., 2002). En la mayoría de las ocasiones es debido a una ausencia emocional momentánea, mientras que en otras está provocado por la realización de tareas de evitación (Bermejo, 2005). Entre los síntomas de este proceso se suele distinguir entre angustia por separación y duelo traumático. El primero de ellos es el núcleo de un duelo complicado, relacionado con el apego. El segundo representa manifestaciones del duelo relacionadas con el trauma provocado por la muerte, entre las que se pueden encontrar sentimientos de falta de propósito sobre el futuro, sensación de entumecimiento, sensación de shock o aturdimiento, dificultad para reconocer la muerte, sentir que la vida está vacía o sin sentido, falta de confianza o seguridad, o ira por la muerte (Dodd et al., 2008). La última edición del Manual Diagnóstico y Estadístico de los Trastornos Mentales (DSM-5) incluye el "trastorno persistente de duelo complicado" para referirse a la incapacitación causada por la pena, la focalización del pensamiento de la persona en la pérdida y el escaso o nulo interés por actividades o relaciones (APA, 2OI3), lo que puede manifestarse en forma de autorreproches, irritabilidad, aislamiento social, enlentecimiento psicomotor acusado, abuso de sustancias psicoactivas y trastornos del sueño, entre otras alteraciones (Uribe, 20I 5). El DSM-5 incluye así el duelo como un precipitante que puede llevar a un episodio depresivo mayor, contribuyendo a la polémica por psicopatologizar una reacción normal ante la pérdida, facilitar la medicalización y apurar los tiempos y las formas que cada persona necesita para elaborar el duelo (Wakefield, 20I3) 
Patológico o no, parece lógico pensar que el proceso de duelo pueda tener una serie de peculiaridades en sus manifestaciones en las personas con DI. Así, sus limitaciones en el funcionamiento intelectual podrían dificultar el procesamiento de los eventos adversos que aparecen en su vida, lo que obstaculizaría el afrontamiento de un episodio tan complicado como el duelo (Mevissen et al., 20I6). Interesa por tanto, entre otras muchas cuestiones relativas a esta temática, conocer cuáles son las características de este proceso en el colectivo de personas con DI, cuál es su comprensión del concepto de muerte, qué cambios aparecen cuando sufren la pérdida de una persona significativa, en qué medida pueden ser más vulnerables a desarrollar un duelo complicado o atípico y van a requerir apoyos, cuál es la respuesta social que reciben ante esta situación y cuáles son las necesidades derivadas de la pérdida. Esta información, más que para calificar sus procesos de duelo como "patológicos", resulta relevante para el diseño e implementación de apoyos eficaces para ayudarles a superar la pérdida y afrontar los cambios que de ella se deriven.

En respuesta a estos planteamientos, los objetivos de este trabajo se centran, en primer lugar, en determinar cuáles son las características (diferenciales o no) que acompañan al proceso de duelo en la población con DI; en segundo lugar, conocer y examinar los instrumentos específicos existentes para evaluar este proceso de duelo en personas con DI; y en tercer lugar, revisar los tipos de intervención dirigidos a proporcionar los apoyos más adecuados a sus necesidades cuando se enfrentan a un proceso de duelo.

\section{Método}

- Tipo de estudio

Se realizó un estudio de revisión agregativo sobre DI y duelo con el fin de unificar y relacionar los resultados de los estudios más recientes y relevantes, así como identificar las posibles lagunas sobre el tema (Fernández-Ríos y Buela-Casal, 2009).

\section{- Procedimiento}

Para la realización de este trabajo se llevó a cabo una búsqueda en las bases de datos Pubmed, Scopus, PsycInfo, Web of Science, Dialnet y Google Scholar. El término de búsqueda utilizado fue discapacidad intelectual (i. e., 'intellectual disability', 'learning disabilities' y 'mental retardation') combinado con duelo (i. e., 'bereavement'). A continuación, se realizaron cuatro búsquedas más añadiendo a las palabras anteriores los términos evaluación, herramienta, intervención y terapia (i. e., 'assessment', 'tool', 'intervention' y 'therapy'). La búsqueda se limitó a los artículos publicados, en inglés y en español, entre enero de 2007 y febrero de 2017. No obstante, las referencias en estos artículos a otros más antiguos de especial relevancia llevaron a incluir algunas referencias menos actuales por su especial interés y trascendencia.

\section{- Materiales}

Inicialmente las palabras clave utilizadas arrojaron un resultado de 80 referencias en la base de Pubmed, 66 en Scopus, I 57 en PsycInfo, I 52 en Web of Science, 4 en Dialnet ( 3 en español y I en inglés) y más de 40.000 referencias en Google Scholar, por lo que se usó este portal solo para búsquedas más concretas. Para la selección de los artículos se estudiaron los títulos y palabras clave de las publicaciones. Así, se seleccionaron un total de 72 referencias, las cuales se distribuyen cronológicamente como se muestra en el Gráfico I, en la que se puede apreciar que los años con mayor productividad científica sobre este tema fueron 2008, 20I3 y 2016 . El 94.36\% fueron artículos en inglés, siendo solo cuatro los trabajos redactados en español. Por otro lado, los autores con mayor número de publicaciones fueron McEvoy $(n=9)$, Dodd $(n=6)$ y Guerin $(n=6)$. 


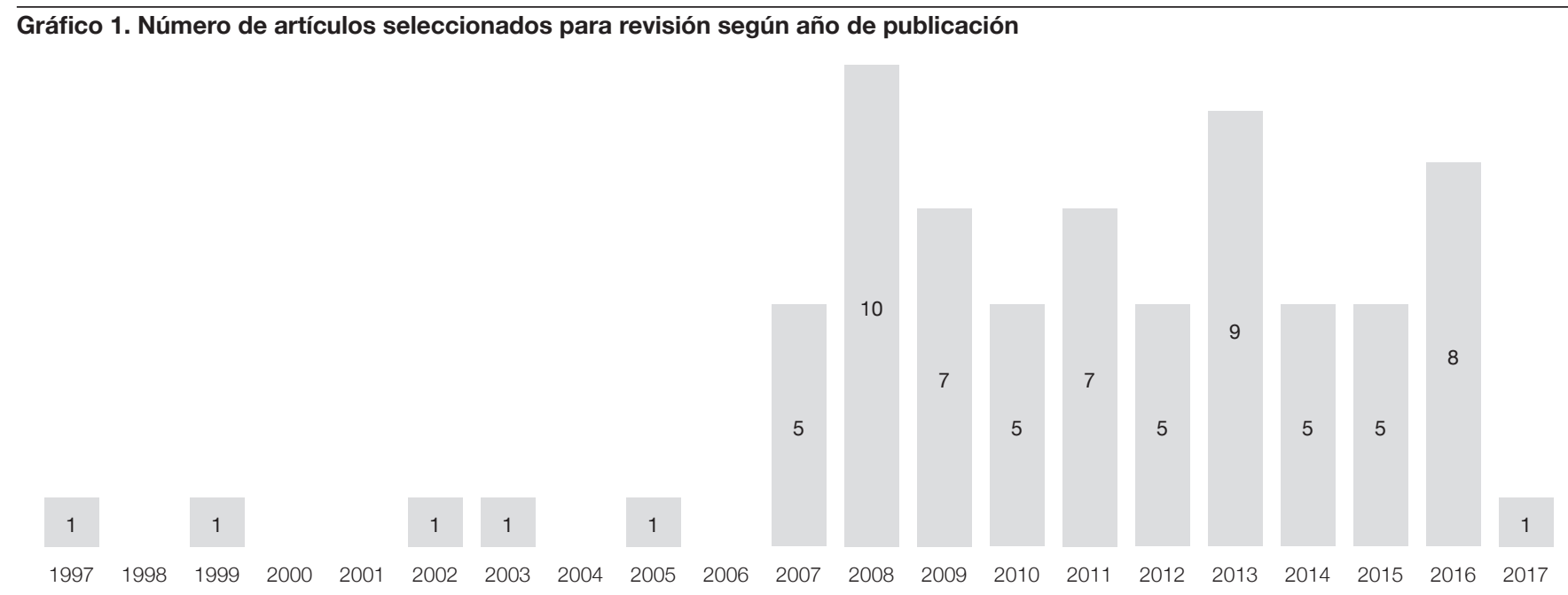

Fuente: elaboración propia.

\section{- Análisis de la información}

El material se organizó en torno a los tres objetivos planteados y, en cada uno de ellos, siguiendo un orden cronológico. Así, se analizaron los estudios en función de su temática y actualidad. Tras la lectura exhaustiva de los diferentes trabajos, se elaboró una síntesis de los aspectos más relevantes relacionados con el proceso de duelo en la población con DI.

\section{Resultados}

- Características del proceso de duelo en personas con DI

En la Tabla I se recogen los estudios que abordan las características del proceso de duelo en personas con DI. Parece lógico pensar que estas personas entiendan la muerte de una manera diferente debido a la carencia de información y las dificultades cognitivas, pero no se les puede negar la existencia de malestar y sufrimiento (Blackman, 20I6). En las sociedades occidentales la muerte es con frecuencia un tema tabú y se insta a minimizar las emociones negativas (LoConto y Jones-Pruett, 2008), provocando un aumento en la patologización del duelo. Si a ello añadimos que a las personas con DI no se les suele informar de la pérdida, que tienen pocos espacios para hablar de su tristeza, que no se tolera de igual forma su sufrimiento y utilizan en ocasiones estrategias inadecuadas para el afrontamiento del dolor, la situación se cronifica aún más y puede dar lugar a conductas más desajustadas. La sobreprotección a la que a menudo se ven expuestos, además de mermar sus capacidades, crea un entorno alejado de la realidad. Esta manera de actuar es tranquilizadora para quienes les rodean pero, al mismo tiempo, les limita la comunicación y el contacto con su entorno (Garvía-Peñuelas, 2009).

McRitchie et al. (20I4) afirman que este colectivo experimenta el duelo y el dolor de forma similar a la población general. Se debe entender este proceso como un momento emocional intenso que provoca dolor y angustia, no como una enfermedad ni un trastorno ansioso-depresivo, aunque en algunas ocasiones los cambios cognitivos y comportamentales puedan apuntar en esta dirección (Shear, 20I2). $\mathrm{Y}$ es que, dado que las personas con DI no utilizan las mismas estrategias interpretativas 
que el resto de la población, es posible que no lloren de inmediato o que comuniquen su pena a través de comportamientos que no se interpretan fácilmente como conductas de duelo (LoConto y Jones-Pruett, 2008), lo que puede dar lugar a creencias erróneas sobre la existencia de algún otro tipo de trastorno.

El primer estudio sistemático centrado en la exploración del proceso en adultos con DI (Hollins y Esterhuyzen, I 997), ya concluye que se había subestimado el impacto del duelo en términos de morbilidad psiquiátrica y conductual. Posteriormente, Bonell-Pascual et al. ( I999) realizaron un estudio longitudinal en el que se observó que este colectivo mostraba una respuesta al duelo muy similar en tipo al resto de la población, y solo difería en la expresión del mismo. La DI resultó ser un predictor significativo de problemas de salud mental después de la pérdida de un ser querido. Años más tarde, Esbensen et al. (2008) concluían que la muerte de uno de los padres no iba seguida de cambios en las habilidades funcionales, pero sí estaba asociada a alteraciones de conducta que aparecían habitualmente tras un lapso de tiempo desde la notificación de la muerte (Morgan y McEvoy, 20I4). Entre dichos cambios destacaban la hiperactividad, la letargia y la irritabilidad (Brickell y Munir, 2008), así como la 'handicapped smile' o sonrisa de las personas con DI, de la que ya hablaba Sinason en I992 (citado por Boyden et al., 20I0), que consiste en un mecanismo que utilizan en ocasiones para adaptarse a las demandas de la sociedad mediante la ocultación de su dolor.

Blackman (2016) agrupa los factores implicados en el duelo en esta población en tres tipos: los derivados directamente de la DI, el impacto de la discapacidad en el apego y los factores ambientales. Con respecto al primero, la propia DI, se relaciona de forma directa con la comprensión del concepto de muerte. En el último estudio de McEvoy et al. (20I7) se entrevistó a 30 personas con DI con el fin de evaluar el conocimiento de las funciones vitales de los seres humanos y la comprensión del concepto de muerte, pues ambas concepciones se consideran relacionadas. La comprensión puede variar en los adultos desde el conocimiento parcial hasta un completo entendimiento en función de diversos factores, entre los que destacan las habilidades cognitivas y adaptativas (McEvoy et al., 201 2; McEvoy et al., 2017). Se cree que los sujetos con niveles más graves de discapacidad tienen reducida esta capacidad de comprensión (Young, 20I6) y, por ende, sienten una angustia más intensa, son más vulnerables a tener pensamientos erróneos y manifiestan un nivel de miedo a la muerte más alto (McEvoy et al., 20I 2; Stancliffe et al., 2016). Por tanto, ayudarles a entender el concepto de muerte ayudaría también a comprender mejor las consecuencias, los procesos y los sentimientos involucrados en el duelo y facilitaría la provisión de apoyos adecuados para evitar la cronificación del mismo (Rodríguez Herrero et al., 201 5 ).

En lo concerniente al impacto de la discapacidad en el apego, cabe subrayar la frecuencia con que las personas con DI no son informadas del deceso. Las razones principales por las que no se les comunica ni se les habla sobre la muerte suelen ser tres (LoConto y Jones-Pruett, 2008; Wiese et al., 201 5). La primera hace referencia a sus limitaciones en el funcionamiento intelectual: ¿para qué informales si no pueden comprender la muerte puesto que consta de componentes abstractos? La segunda se fundamenta en el hecho de negar su capacidad de sufrimiento: ¿para qué tratar de explicarles nada si no están sufriendo? En tercer lugar, están las actitudes y conductas de sobreprotección hacia la persona: ¿no será mejor evitar angustia a la persona con DI y a quien debe comunicarle la noticia, quien tampoco sabe bien cómo afrontar el tema? Sin embargo, no recibir la noticia de la muerte puede tener consecuencias negativas en el vínculo de apego y en su autoestima, ya que puede sentirse abandonada por un ser querido que ha desaparecido de su vida sin razón aparente.

En cuanto a los factores ambientales, existen evidencias relacionadas con una sintomatología del duelo menos intensa (que incluso cesa) si se les proporcionan los apoyos necesarios (Campbell y Bell, 20I0). De hecho, se han llevado a cabo estudios que demuestran los 


\begin{tabular}{|c|c|c|c|c|}
\hline Autor/es & Año & $\begin{array}{l}\text { Tipo de } \\
\text { estudio }\end{array}$ & Muestra & Instrumentos \\
\hline Hollins y Esterhuyzen & 1997 & Empírico & $\begin{array}{l}100 \text { personas con DI ( } 50 \text { con } \\
\text { duelo y } 50 \text { sin duelo) }\end{array}$ & $\begin{array}{l}\text { - } \text { Cuestionario semiestructurado de duelo } \\
\text { - Aberrant Behaviour Checklist (ABC) } \\
\text { - Psychopatology Instrument for Mentally Retarded } \\
\text { - } \text { Adults (PIMRA) } \\
\text { - Life Events Checklist } \\
\end{array}$ \\
\hline Bonell-Pascual et al. & 1999 & Empírico & $\begin{array}{l}41 \text { personas en duelo con } \\
\text { DI de los } 50 \text { participantes } \\
\text { del trabajo de Hollins y } \\
\text { Esterhuyzen } \\
\end{array}$ & $\begin{array}{l}\text { - Aberrant Behaviour Checklist (ABC) } \\
\text { - Psychopatology Instrument for Mentally Retarded } \\
\text { Adults (PIMRA) }\end{array}$ \\
\hline Brickell y Munir & 2008 & Teórico & - & - \\
\hline Dodd et al. & 2008 & Empírico & $\begin{array}{l}76 \text { personas con DI: } \\
36 \text { habían experimentado } \\
\text { duelo en los dos años previos. } \\
36 \text { sin duelo en los dos años } \\
\text { anteriores }\end{array}$ & $\begin{array}{l}\text { - Complicated Grief Questionnaire for People with } \\
\text { Intellectual Disabilities (CGQ-ID) } \\
\text { - } \quad \text { Bereavement History Questionnaire } \\
\text { - Index of Social Competence }\end{array}$ \\
\hline Esbensen et al. & 2008 & Empírico & $\begin{array}{l}390 \text { adultos con DI: } 150 \text { con } \\
\text { síndrome de Down y } 240 \text { con } \\
\text { otro tipo de DI }\end{array}$ & $\begin{array}{l}\text { - Versión revisada de Barthel Index (30 ítems de } \\
\text { - evaluación de habilidades funcionales) } \\
\text { - Inventory for Client and Agency Planning } \\
\text { - (ICAP), para problemas de conducta }\end{array}$ \\
\hline LoConto y Jones-Pruett & 2008 & Teórico & - & - \\
\hline Ruiz y Sainz & 2008 & Teórico & - & - \\
\hline Dodd y Guerin & 2009 & Teórico & - & - \\
\hline Boyden et al. & 2010 & Empírico & 5 personas con DI en duelo & $\begin{array}{l}\text { - Cuestionarios accesibles de feedback de la } \\
\text { terapia de grupo }\end{array}$ \\
\hline Campbell y Bell & 2010 & Empírico & $\begin{array}{l}\text { Una persona con DI leve en } \\
\text { duelo }\end{array}$ & $\begin{array}{l}\text { - Conceptions of Death Questionnaire } \\
\text { - Concept of Living and Death Questionnaire } \\
\text { - The Understanding Emotions Questionnaire }\end{array}$ \\
\hline McEvoy et al. & 2012 & Empírico & 34 personas con $\mathrm{DI}$ & - Concept of Death Questionnaire (CODQ) \\
\hline Shear & 2012 & Teórico & - & - \\
\hline Forrester-Jones & 2013 & Empírico & $\begin{array}{l}40 \text { cuidadores y } 26 \text { personas } \\
\text { con y } \sin \mathrm{DI}\end{array}$ & $\begin{array}{l}\text { - Entrevista semiestructurada en torno a los } \\
\text { funerales, organización y apoyo prestado. }\end{array}$ \\
\hline McRitchie et al. & 2014 & Empírico & 13 adultos con DI & $\begin{array}{l}\text { - Entrevistas semiestructuradas utilizando análisis } \\
\text { fenomenológico interpretativo }\end{array}$ \\
\hline Morgan y McEvoy & 2014 & Empírico & 10 cuidadoras principales & $\begin{array}{l}\text { - Entrevistas semiestructuradas utilizando análisis } \\
\text { fenomenológico interpretativo }\end{array}$ \\
\hline Blackman & 2016 & Teórico & - & - \\
\hline Stancliffe et al. & 2016 & Empírico & $\begin{array}{l}39 \text { personas con DI } \\
40 \text { cuidadores }\end{array}$ & $\begin{array}{l}\text { - } \quad \text { Inventory for Client and Agency Planning (ICAP) } \\
\text { - } \quad \text { Concept of Death Questionnaire (CODQ) } \\
\text { - } \quad \text { Fear-of-Life Planning Scale (EOLPS) } \\
\end{array}$ \\
\hline Young et al. & 2016 & Empírico & 7 cuidadores & $\begin{array}{l}\text { - Entrevistas semiestructuradas utilizando un } \\
\text { análisis fenomenológico interpretativo }\end{array}$ \\
\hline McEvoy et al. & 2017 & Empírico & 30 adultos con DI & $\begin{array}{l}\text { - The British Picture Vocabulary Scale-II } \\
\text { - The Index Social Competence } \\
\text { - The Body Interview } \\
\text { - Death Interview }\end{array}$ \\
\hline
\end{tabular}

Nota: DI= discapacidad intelectual.

Fuente: elaboración propia. 
beneficios de incluir a las personas con DI en las actividades y procesos relacionados con la muerte y el duelo, como la asistencia al funeral, al tanatorio y a otros rituales (Gilrane y Targgart, 2007). Sin embargo, los datos que recogen el grado de participación en estos son muy variables. Mientras que en el trabajo realizado por Dodd et al. (2008), se observa que el $95 \%$ de los participantes habían tenido la posibilidad de asistir al funeral y el $90 \%$ pudieron hablar sobre el fallecido y observar fotografías de la persona amada, en el estudio de Forrester-Jones (20I3) el $60 \%$ señalaba no haber acudido al funeral y haber sido informado de éste una vez que ya había tenido lugar. Parece que nos encontramos ante una conspiración de silencio que estaría favoreciendo la pérdida de autonomía de esta población (ForresterJones, 2013; Read y Elliott, 2007), pues no sólo se les oculta la verdad, sino que se les ha negado la posibilidad de que elaboren su propio proceso de duelo, reprimiendo sentimientos y proporcionándoles en muchos casos información errónea para evitarles dolor (Ruiz y Sainz, 2008).

No obstante, también se ha señalado una correlación positiva entre implicarse mucho en los rituales de la muerte y el desarrollo de un duelo complicado, lo que parece indicar que, si bien es importante que las personas se involucren en estos rituales, la exposición previa a los mismos y el desarrollo de la comprensión sobre el proceso son pasos precedentes esenciales para evitar, por ejemplo, que la asistencia al funeral desborde a la persona (Dodd et al., 2008). En esta línea, la educación para la muerte puede tener un papel central pues, como ya se ha mencionado, la comprensión de la misma parece ser un factor que favorece la elaboración de un duelo normalizado. McRitchie et al. (20I4) señalan que han de tenerse en cuenta otros aspectos que interfieren en el proceso de afrontamiento del duelo y que deberían ser abordados de forma previa a la pérdida, en concreto: (a) las experiencias intra e interpersonales (i.e., respuesta emocional, pérdidas y exclusión); (b) sus creencias sobre la vida y la muerte; (c) el nivel de inclusión; y (d) la relación con el difunto.
Además, debe tenerse en cuenta la existencia de una multiplicidad de pérdidas secundarias (que siguen a la pérdida principal del ser querido y que estarían influyendo en el duelo de la persona con DI) como pueden ser el cambio de domicilio, amigos, mascotas o rutinas, entre otras (Brickell y Munir, 2008; Galán, 20I4). A pesar de su importancia, estas pérdidas secundarias frecuentemente pasan desapercibidas, cuando son estas las únicas pérdidas que en muchas ocasiones pueden ser mitigadas, pues la muerte es irreversible, pero ante la pérdida de amigos por un cambio de domicilio, por ejemplo, sí se pueden organizar actividades que permitan disminuir el sufrimiento (Brickell y Munir, 2008).

Todas estas particularidades del proceso de duelo en la población con DI permiten concluir que, aunque este puede presentar un curso no patológico, sí existe una mayor vulnerabilidad y un mayor riesgo de sufrir una cronificación del mismo. De hecho, se observa en muchas ocasiones que el patrón de cambio conductual se mantiene o empeora en períodos superiores a los dos años (Brickell y Munir, 2008; Dodd y Guerin, 2009; Esbensen et al., 2008). En la línea de lo expuesto por Brickell y Munir (2008), entre las posibles explicaciones a esta complicación del proceso se encontrarían: las pérdidas secundarias, las barreras en la comunicación efectiva y las dificultades para la comprensión de la pérdida.

- Herramientas de evaluación del duelo en personas con DI

La literatura científica en torno a los instrumentos de evaluación que los profesionales pueden emplear en el proceso de duelo en el colectivo con DI es escasa. La mayoría de las herramientas se han desarrollado ad hoc con fines investigadores, no generalizándose su uso más allá de los estudios. Además, lo más habitual es el empleo de entrevistas o de pequeños cuestionarios desarrollados para la población general. El análisis de las referencias seleccionadas va a girar, por un lado, sobre el tipo de entrevista utilizada en los diferentes trabajos y, de otro, sobre los cuestionarios empleados en el campo del duelo en DI. 
Así, las entrevistas semiestructuradas han sido las herramientas más utilizadas en un buen número de investigaciones. Estas han permitido evaluar diferentes aspectos relacionados con la muerte y con la historia de vida de la persona, aportando más información que un inventario. En algunas ocasiones, se han realizado a la propia persona con DI (McEvoy et al., 20I2), mientras que en otras se obtiene la información a partir de terceros, normalmente de sus cuidadores y proveedores de apoyos principales (Clute y Kobayachi, 20I 2; Morgan y McEvoy, 20I4; Young et al., 2016).

En cuanto a los cuestionarios, se seleccionaron aquellos desarrollados específicamente para nuestra población de interés (Tabla 2) a pesar de que, como señalábamos anteriormente, aparece en la literatura revisada la utilización de escalas de la población general para evaluar determinados aspectos. En orden cronológico, encontramos en primer lugar los tres instrumentos utilizados en la investigación de Mappin y Hanlon (2005). El primero de ellos, The Death Concept Questionnaire, consta de I4 ítems, de respuesta dicotómica (sí/no), que evalúan siete componentes: separación, inmovilidad, irreversibilidad, causalidad, no funcionalidad, universalidad e insensibilidad. Se puntúan con I las respuestas correctas y o en el caso contrario. La segunda herramienta utilizada por estos autores fue The Knowledge About Death Questionnaire, que consta de I2 ítems basados en el cuestionario de Yanok y Beifus (I993; citado por Mappin y Hanlon, 2005) en los que se les pide a los participantes que definan diferentes palabras asociadas con la muerte y los rituales que rodean a la misma. En este caso, las respuestas son evaluadas como o, I 02 en función del grado de concreción de la respuesta (Mappin y Hanlon, 2005). La máxima puntuación que pueden obtener es de 24 puntos. En lo que al tercer cuestionario se refiere, The Understanding Emotions Questionnaire (Mappin y Hanlon, 2005), está formado por I 8 ítems que evalúan la comprensión emocional. Mediante el uso de fotografías se hacen a los participantes con DI preguntas cerradas (se les dan tres opciones de respuesta: se siente enfadado/a, feliz o triste) y abiertas (sobre comportamientos y sensaciones físicas asociadas a esas emociones). Las primeras se puntúan como o o I; mientras que las segundas, las abiertas, se puntúan como en el cuestionario anterior (2-I-O). Estas dos últimas escalas mostraron un grado de acuerdo interjueces del $92 \%$ y $94 \%$ respectivamente.

En función de la fecha de publicación del artículo, el siguiente instrumento que encontramos es el 'Bereavement History Questionnaire', adaptación de Dodd et al. (2008) a partir del cuestionario de Hollins y Esterhuyzen (I997). Tiene como objetivo examinar las características del duelo de la población con DI cubriendo las áreas del nivel de contacto con la persona fallecida, las circunstancias en las que se dio la noticia, el nivel de apoyo recibido y el nivel de participación en los rituales. A diferencia de los anteriores, este ha de ser cumplimentado por los profesionales. Los índices de consistencia interna mostrados en términos de alpha de Cronbach (a) para sus dos factores, pre-fallecimiento y postfallecimiento, son $.85 \mathrm{y} .92$, respectivamente.

En ese mismo año, Blackman (2008) desarrolló un cuestionario basado en su experiencia profesional trabajando con personas con DI. El objetivo en este caso no era conocer el proceso de duelo en sí mismo, para su descripción, sino para proporcionar a los profesionales información útil en la provisión de apoyos a las necesidades derivadas del proceso de duelo. Bereavement Needs Assessment Tool (BNAT) consta de ro áreas principales que evalúa a través de 3 I ítems. En este caso, las diferentes preguntas son respondidas por el cuidador. Los resultados obtenidos se podrían organizar en torno a tres áreas de apoyos: cuestiones prácticas, sociales y emocionales.

En cuanto al Complicated Grief Questionnaire for People with Intellectual Disabilities (CGQID), lo hemos introducido en este punto a pesar de haber sido desarrollado previo a los dos cuestionarios anteriores (Dodd, 2007), puesto que sus propiedades psicométricas fueron exploradas en los años posteriores (Dodd et al., 2008; Guerin et al., 2009). Se trata de una 
adaptación del Inventory of Complicated Grief de Prigerson (I999; citado por Guerin et al., 2009) en su versión para niños, seleccionada por la sencillez en su vocabulario y gramática. El objetivo de la escala original es evaluar la pertinencia del diagnóstico de duelo complicado, mientras que en esta adaptación se evalúa la presencia de alguno de los síntomas. Consta, por un lado de I I ítems divididos en dos subescalas principales (ansiedad por separación $n=6$; duelo traumático $n=5$ ) y, por otro, de 4 ítems que no pertenecen a ninguna de las subdimensiones anteriores. El formato de respuesta es escala tipo Likert, con 4 o 5 niveles en función de la cuestión planteada. A su vez, según el enunciado también cambia el formato de respuesta, respondiendo de acuerdo al grado de frecuencia o de cantidad (cuán a menudo o cuánto). En lo que se refiere a las garantías psicométricas, presenta un valor $a$ de .92 para la escala en su totalidad y unos índices adecuados para las dos subescalas principales que lo componen $(a=.86-$ .87) (Guerin et al., 2009).

Otro de los cuestionarios empleados para la población con DI es el Staff Attitude Questionnaire (STAQ) (MacHale et al., 2009). Se trata de una escala que evalúa la percepción de los profesionales acerca de la comprensión del concepto de muerte que tienen las personas con DI con las que trabajan. Se evalúan también las características de la persona sobre la que se cumplimenta, su predicción respecto al grado de severidad de la reacción una vez haya muerto la persona querida, la predicción de la cantidad de apoyo requerida y el nivel de confianza del propio profesional a la hora de reconocer los síntomas. Los diferentes enunciados se encuentran agrupados en seis subcategorías, comprensión cognitiva del concepto biológico de muerte (ítems I, 2, 3, 4), comportamiento disruptivo tras la pérdida (ítem 5 ), necesidad de apoyo extra durante el duelo (ítem 6), confianza del profesional en sí mismo para reconocer los cambios y dar el apoyo necesario (ítems 7,8 ), importancia de las habilidades verbales (ítem 9) y similitud de la duración del proceso con la población sin DI (ítem Io). Finalmente, los datos psicométricos informados por los autores reflejan un valor $a$ de .70 para la escala en su conjunto y de .84 para los cuatro ítems que evalúan la comprensión del concepto biológico de muerte.

\section{The Lancaster and Northgate Trauma Scales}

(LANTS) (Wigham et al., 20I I) es otra herramienta desarrollada con el fin de evaluar los eventos vitales traumáticos en las personas con DI. Consta de 29 ítems autoinformados y 43 ítems cumplimentados por el cuidador principal. Los análisis psicométricos muestran unos valores adecuados de consistencia interna tanto en la escala autoinformada ( $a$ : .84) como en las contestadas por los informadores (informant behavioral changes subscale a: .82; frequency subscale a: .80; y severity subscale $a$ : $.84)$. En cuanto al análisis de las evidencias de validez, se analizó la correlación de Spearman entre LANTS y otras medidas previamente establecidas del trauma, encontrando resultados satisfactorios.

\section{Por último, The Concept of Death}

Questionnaire (CODQ) (McEvoy et al., 20I 2) es una escala autoinformada de $\mathrm{I} 3$ ítems para las personas con DI en duelo que evalúan la comprensión del concepto de muerte a través de cinco componentes (i.e., finalidad/ irreversibilidad, universalidad, no-funcionalidad, inevitabilidad y causalidad). A los participantes se les preguntaba acerca de tres viñetas, presentadas en orden aleatorio, relacionadas con la muerte. Su formato se basa en el desarrollado por Mahon, Goldberg y Washington (I999; citado por McEvoy et al., 20I2). Las preguntas podían ser en algunos casos respondidas con sí/ no (i.e., ¿alguna vez estará vivo de nuevo?) o con una respuesta abierta (i.e., ¿qué hace que la gente muera?). Todas ellas se valoran de o a 2 en función de la no comprensión (o), comprensión parcial (I) y comprensión consciente (2). De esta forma, las puntuaciones más altas indicarían una mejor comprensión. En lo que refiere a las garantías psicométricas, en el estudio de McEvoy et al. (2OI2), se obtuvo un valor $a$ de $.75 \mathrm{y}$ un acuerdo interjueces de .87. Años más tarde, en el trabajo de Stancliffe, Wiese, Clayton, Read y Jeltes (20I 5 ; citado por Stancliffe et al., 20I6) se encontró un valor a de .80 para los adultos con DI y un $a$ de .99 en los cuidadores, además de un acuerdo interjueces del 100\%. 


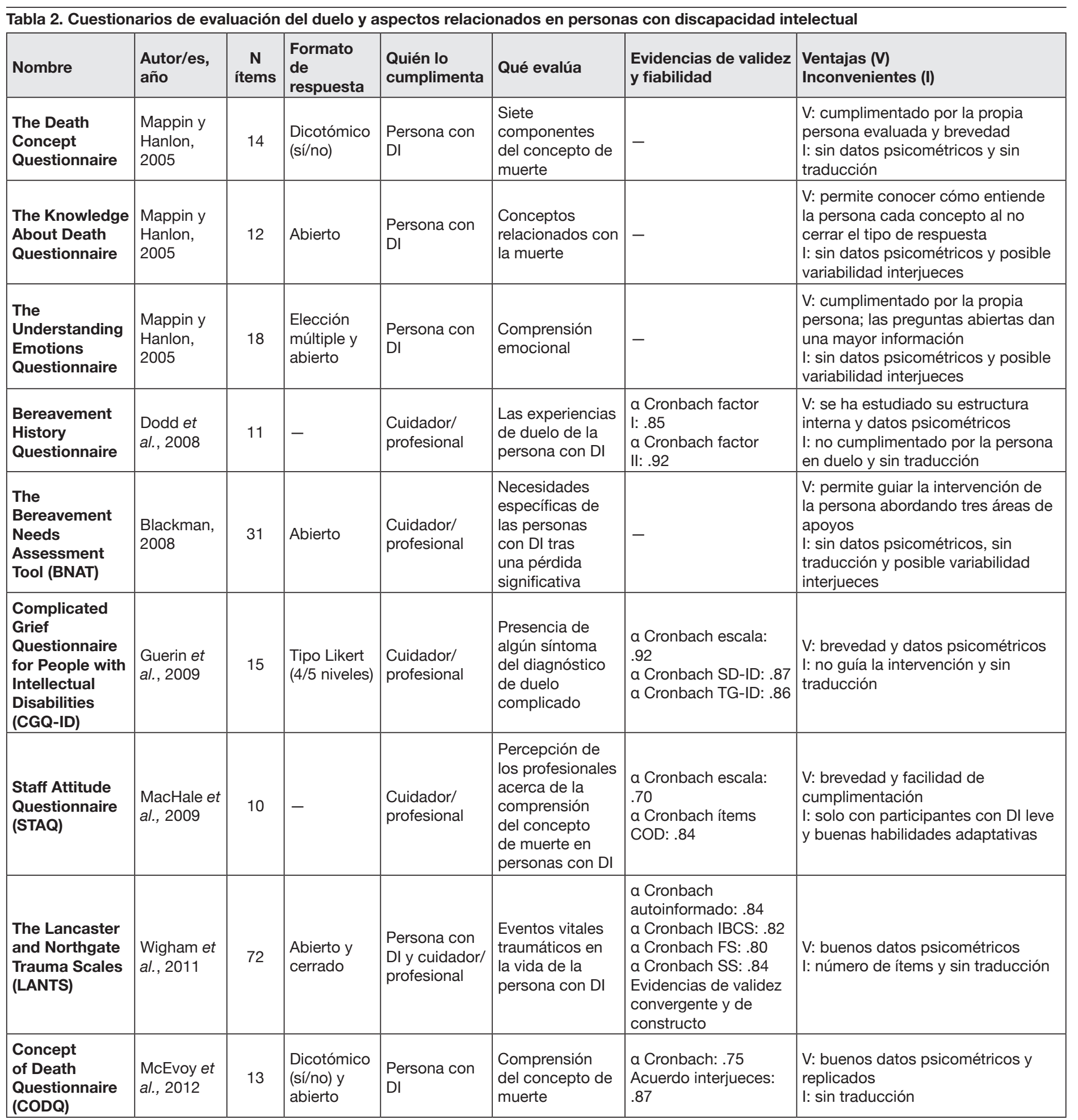

Nota. DI= discapacidad intelectual; $\mathrm{SD}-\mathrm{ID}=$ Separation distress - intellectual disabilities; TG-ID= Traumatic grief - intellectual disabilities; $\mathrm{COD}=$ compression of death; IBCS= informant behavioral changes subscale; FS= frequency subscale; $S S=$ severity subscale.

Fuente: elaboración propia. 
- Intervención y tratamiento en el proceso de duelo en DI

Las técnicas de intervención más utilizadas por el momento se podrían resumir en dos grandes líneas. Por un lado, se están utilizando los grupos como un espacio en el que la persona se encuentra en un entorno seguro que le facilita la comunicación con sus iguales y que le abre la puerta a un tema conversacional complejo, sobre el que apenas tiene conocimientos y sobre el que su entorno más inmediato no suele hablar (Boyden et al., 20Io). Por otro lado, se está fomentado el uso de la psicoterapia como una forma de ayudar a la persona en duelo una vez que este proceso ha comenzado a complicarse o que la persona se ha quedado sin estrategias de afrontamiento, incurriendo también en la cronificación del duelo. Dichas intervenciones, ya sea individualmente o en grupo, deben ser planificadas de acuerdo a la comprensión de la persona, los síntomas presentados y el sistema de apoyo con el que cuenta (Sttodart et al., 2002).

Si nos centramos en las terapias individuales clásicas encontramos que se han empleado casi todas ellas. En lo que se refiere a la terapia cognitivo-conductual, basada en un enfoque centrado en el presente, su evidencia de fiabilidad en este colectivo y para esta temática es limitada (Brown et al., 20I I). La dificultad a la hora de emplear esta técnica radica en las limitaciones en el funcionamiento intelectual y las habilidades de comunicación oral que puede tener la persona con DI. Esto mismo ocurre con el asesoramiento psicológico, puesto que se trata de un tipo de intervención que colocaría la responsabilidad del tratamiento en el paciente con el fin de aumentar la autoestima y reducir la inseguridad (Brown et al., 20I I), pero en muchas ocasiones estas personas no disponen de un insight suficientemente elaborado como para reconocer las carencias sobre las que se ha de trabajar. En cuanto a la terapia psicodinámica, autores de prestigio como Blackman (20I6) la señalan como una de las mejores alternativas ante los casos de duelo complicado pero, como ocurría con las terapias anteriores, no contamos con las evidencias suficientes que permitan apoyar la mayor eficacia de esta forma de intervención. No obstante, algunos estudios muestran una reducción de conductas problemáticas independientemente del grado de discapacidad (Parkes et al., 2007). Finalmente, debido al papel tan relevante que juegan las familias en esta población, podría ser más acertado el empleo de otras terapias tales como la terapia sistémica o familiar. Siguiendo este enfoque, Baum y Walden (2006; citado por Brown et al., 20I I) describieron el proceso de creación de un servicio que contaba con este tipo de psicoterapia para personas con DI en Londres. Los resultados obtenidos por estos profesionales mostraron que se trataba de un proceso terapéutico lento, cuya duración oscilaba desde los I 2 a los I7 meses.

Independientemente del tipo de terapia empleado, es necesario un estudio del cuerpo humano desde un enfoque vitalista que permita a la persona entender y tener un mejor conocimiento del concepto de la muerte (McEvoy et al., 20I 2; 20I7). Así pues, un modelo de soporte holístico permitirá integrar los sistemas de cuidado (Read y Elliot, 2007). De esta forma, estudios de caso como el de Hannah (Campbell y Bell, 20I0) muestran, gracias a una intervención en el contexto vital y utilizando los recursos del medio, beneficios en la normalización del duelo en un período de tres meses. Es importante tener en cuenta que en este caso también se ha trabajado el entrenamiento con la familia. Por tanto, no se debe olvidar la necesidad de contar con profesionales y familiares bien formados y sensibles a esas peculiaridades del proceso de duelo de la persona a la que se está dando soporte y apoyo.

Si nos centramos en el empleo de los grupos como una forma de abordar el tema del duelo en DI encontramos que, a pesar de que la literatura científica indica que por lo general estas terapias grupales no han tenido un foco central, existen varios trabajos que evalúan los efectos positivos de estas intervenciones. Así, en el trabajo de Sttodart et al. (2002) se trató de comprobar si los participantes se beneficiaban de un programa de este estilo utilizando para ello medidas de depresión, ansiedad y conocimiento de la muerte 
antes y después del programa. Los resultaron reflejaron que las puntuaciones en depresión fueron significativamente menores, mientras que las referidas a ansiedad y comprensión de la muerte no mejoraron.

Años más tarde, Boyden et al. (2010) y Borsay et al. (2013) estudiaron el empleo de la terapia grupal en un grupo con cinco y cuatro personas en duelo, respectivamente, en un programa de ocho semanas consecutivas. Se recabó feedback cualitativo a través de las experiencias reportadas por los participantes (i.e., referían haber aprendido a manejar "sentimientos difíciles") y cuantitativo mediante escalas de depresión de la población general y la Clinical Outcomes in Routine EvaluationLearning Disability (CORE-LD) que evalúa el funcionamiento cotidiano del colectivo con DI, encontrando cambios positivos en tres cuartas partes de los participantes (Borsay et al., 20I3).

Esta intervención grupal ha ido introduciendo terapias más actuales como la musicoterapia. Holey y McKinney (2015) emplearon un tratamiento de nueve semanas con tres adultos con DI. El objetivo era educar en cuestiones relacionadas con la muerte, así como las estrategias para afrontar los sentimientos que surgen cuando una persona significativa muere. No se encontraron evidencias que indicaran un valor añadido de este tipo de terapias frente a las comúnmente utilizadas.

Se hace especial hincapié en que todas las intervenciones incluyan enfoques psicoeducativos y psicoterapéuticos, con el doble objetivo de asegurar que los participantes estén informados y comprendan los conceptos básicos de la muerte, y que a su vez cuenten con un espacio para explorar sus propias experiencias. Aunque, desafortunadamente, hay una falta de medidas estandarizadas disponibles para evaluar cuantitativamente la efectividad de estas intervenciones (McEvoy et al., 20I2; McRitchie et al., 2OI4).

Por otra parte, es importante también que estas estén dirigidas al entorno más inmediato de la persona, puesto que como señalábamos con el caso de Hannah (Campbell y Bell, 20I0), los mejores resultados se obtienen cuando se trabaja con el contexto en su conjunto. Así, se están diseñando nuevos programas educativos que ponen especial interés en la necesidad de formar a los profesionales y familiares proveedores de apoyos (Rodríguez Herrero et al., 20I3 b). Esta tendencia surge como respuesta a la urgente necesidad de asesorar a los profesionales que no en pocas ocasiones son los únicos testigos del desarrollo traumático del duelo y de las elevadas demandas emocionales por parte de las personas con DI (Gilrane y Taggart, 2007; Gray y Abendroth, 2016; Handley y Hutchinson, 20I3; Morgan y McEvoy, 20I4). En esta línea, se evaluó el impacto de un programa de entrenamiento para los profesionales en conocimiento y confianza en el soporte a las personas con DI durante los procesos de duelo. Los resultados obtenidos en una muestra de 33 cuidadores vislumbraron un aumento en la confianza que, a pesar de no ser significativo, tuvo un impacto en el soporte proporcionado y en su satisfacción laboral (Reynolds et al., 2008). Watters et al. (20I2), en un estudio similar, evaluaron la formación de 48 cuidadores obteniendo un incremento significativo en el conocimiento sobre el duelo. En esta ocasión el entrenamiento consistió en un curso de capacitación de seis horas de duración y cuyos contenidos se centraron en: (a) el luto y el duelo; (b) dificultades para el duelo y el aprendizaje; y (c) apoyo a la persona con DI a través del proceso de la pérdida (i.e., preparación para el duelo, apoyo práctico tras el mismo, facilitación del proceso, identificar dificultades y cuidar los recursos disponibles). Planteamientos formativos de este tipo dirigidos a los profesionales permiten que, en aquellos momentos en los que no se sientan capacitados para dar respuesta a alguna de las demandas de la persona con DI que ha sufrido una pérdida, el asesoramiento por parte de otros profesionales expertos favorezca la superación con éxito del problema (Herrán y Cortina, 2008a). De esta forma, el profesional se siente más seguro y percibe un mayor sentimiento de autoeficacia del profesional a la vez que se evita un estancamiento de la problemática por un conflicto no superado en el proceso de elaboración del duelo. 
Recientemente, Gray y Truesdale (2015) han puesto de relieve la necesidad de capacitar a los profesionales que se encuentran en el microsistema de las personas con DI para que estén preparados para hacer frente a las necesidades derivadas del proceso de duelo. Es necesario que conozcan en profundidad el tipo de contenidos e información que deben proporcionar a estas personas así como las estrategias más adecuadas para aportarles dicha información. Dado que los problemas y las limitaciones en la comunicación impiden en muchas ocasiones que las personas con DI reciban los apoyos necesarios, en los últimos años se está trabajando en el desarrollo de nuevas formas de actuación que potencien su bienestar emocional y psicológico. Para ello, se han desarrollado herramientas de facilitación emocional como la 'memory box' y softwares que permiten desbloquear ciertos temas conversacionales y expresar y manifestar a la persona el dolor con su cuidador (Read et al., 20I3; Young y Garrard, 20I 5 ).

Finalmente, es preciso señalar la importancia y necesidad de prácticas que faciliten el afrontamiento del proceso de duelo dentro de los sistemas educativos. Si bien el funcionamiento ideal debiera integrar programas preventivos dentro del marco educativo, en los últimos años se han desarrollado estrategias que permiten un enfoque de intervención integral. En esta línea, una de las técnicas empleadas es el “acompañamiento educativo" (Herrán y Cortina, 2008b; Rodríguez Herrero et al., 20I3c), basada en una metodología de trabajo no directiva con personas con DI de cualquier edad que han sufrido la pérdida de una persona significativa. El objetivo fundamental es establecer canales de comunicación con el niño y posibilitar que se apoye en el profesional o cuidador cuando lo necesite. Una de las características más relevantes de esta técnica es seguir el proceso de duelo dejando que sean las propias necesidades de la persona con DI las que guíen y proporcionen una jerarquía de intervención (Herrán y Cortina, 2008b; Rodríguez Herrero et al., 20I3c). No obstante, por el momento aún se precisa de una mayor investigación sobre la eficacia de las intervenciones mencionadas en este apartado.

\section{Discusión y conclusiones}

Como se ha puesto de manifiesto a lo largo de esta revisión, la investigación del duelo en las personas con DI es aún muy incipiente. Por esta razón, con el propósito de clarificar los últimos avances en el campo, así como las carencias de estudio existentes, se han propuesto tres objetivos que se corresponden con tres grandes líneas de investigación relacionadas con el tema. Por un lado, un primer objetivo de este trabajo consistía en determinar las características que acompañan al proceso de duelo en la población con DI. Para ello, se han estudiado con detenimiento todos aquellos textos seleccionados que hacían referencia o aportaban nueva información acerca de las peculiaridades de estas personas en duelo. Si se analizan las características que presenta este colectivo en duelo podemos observar que los cambios predominantes no están relacionados con la experiencia del dolor en sí misma sino con la forma de manejar el mismo (Blackman, 20I6; McRitchie et al., 20I4). Parece que las particularidades estarían causadas por la falta de información, de inclusión y de las estrategias de afrontamiento pertinentes (Forrester-Jones, 20I3; Read y Elliot, 2007; Ruiz y Sainz, 2008). Siguiendo la clasificación tradicional mencionada en la introducción, podríamos señalar que en muchas ocasiones nos encontramos ante duelos complicados que apuntan a la tipología de retardado, pues los cambios que se aprecian en la persona aparecen tras un lapso de tiempo desde su notificación (Morgan y McEvoy, 20I4) y podríamos entender la 'handicapped smile' como una tarea de evitación cuyo objetivo es agradar a los demás, ocultar el dolor y evitar hablar sobre el tema con el entorno más cercano. Aunque, por otro lado, y siguiendo esta misma categorización, también se podría apuntar hacia un duelo crónico, pues como ya mostraron Bonell-Pascual et al. (I999), dos años después de la apreciación de cambios comportamentales y psiquiátricos (Hollins y Esterhuyzen, I997) estos siguen presentes en la vida de la persona, cuando lo habitual es que la sintomatología tenga una 
duración entre seis meses y un año (Echeburúa y Herrán, 2007; Pereira, 2010).

Independientemente de la clasificación utilizada, se debe prestar atención a las particularidades del proceso de cada persona, pues si bien las categorías en algunos casos son importantes (i.e., certificados de discapacidad e investigación) de cara a la intervención resulta más relevante conocer las causas del problema y su topografía. Así, se debe resaltar que, aunque las manifestaciones conductuales no sean como las de la población general, el sufrimiento en el que se ven inmersos es similar (Blackman, 20I6). La mayoría de estas características han sido descritas gracias a trabajos empíricos en los que no solo se obtenía información a partir de los cuidadores, sino que en la mayoría de las ocasiones se entrevistaban y evaluaban a las personas con DI en proceso de duelo. A pesar de los beneficios del empleo de este tipo de participantes, ha de señalarse que son pocos los estudios que han contado con un tamaño muestral suficientemente amplio como para poder realizar inferencias robustas a partir de ellos. Además, salvo excepciones como los trabajos de Hollins y Esterhuyzen (I997) y Bonell-Pascual et al. (I999), no se han realizado estudios longitudinales que permitan observar la evolución del proceso.

Por otro lado, la comparación de las características obtenidas en los diferentes participantes es complicada, pues contamos con muestras muy heterogéneas -Campbell y Bell (2010) utilizaban personas con DI leve, mientras que en otros trabajos no se discernía en función del nivel de DI sino en función de su etiología (i. e., síndrome de Down versus otras etiologías) (Esbensen et al., 2008)- e instrumentos de evaluación muy diversos con escasas garantías metodológicas. Se hace evidente, por tanto, la necesidad de sistematización de los estudios acerca de las características del proceso en este colectivo.

Este punto nos lleva al segundo objetivo de esta revisión, conocer las herramientas de evaluación existentes para el proceso de duelo en el campo de la DI. Como se puede observar en el segundo apartado de resultados, no son pocos los trabajos que emplean herramientas que no han sido diseñadas para este colectivo o que carecen de garantías metodológicas. Existe gran escasez de instrumentos que permitan la evaluación rigurosa del proceso. Aun así, contamos con escalas con buenos resultados de consistencia interna (i. e., LANTS), como es el caso del CGQ-ID (Dodd et al., 2008; Guerin et al., 2009) o el CODQ (McEvoy et al., 2012; Stancliffe et al., 20I6).

Nos encontramos con herramientas destinadas a la evaluación de aspectos muy concretos del proceso, lo que posibilita un mayor número de detalles del mismo. Por ejemplo, el CGQID está evaluando la presencia de síntomas de duelo complicado y el CODQ la comprensión del concepto de la muerte. Así, además de la finalidad original de los cuestionarios, podrían ser utilizados para la valoración de la eficacia de programas o intervenciones. En esta línea, el CODQ podría servir para evaluar programas de adquisición del concepto de muerte, valorando así la eficacia de los mismos con una aplicación pre-programa y otra una vez finalizado el mismo, observando la mejoría en la comprensión del participante con DI. Sin embargo, ninguno de ellos sería útil a la hora de precisar los apoyos necesarios que requiere la persona con la que estamos trabajando, aunque sí aportarían un esbozo de ciertos aspectos del proceso. Parece más interesante el empleo de un cuestionario como el BNAT (Blackman, 2008), diseñado para determinar el tipo de intervención, pues si bien es importante establecer la cronificación del duelo, es aún más relevante señalar sobre qué partes del mismo trabajar y sobre cuáles poder comenzar el proceso de andamiaje.

Otro aspecto destacable es que en poco más de la mitad de las escalas son las propias personas quienes responden. En este sentido, parece más importante la información que nos pueda proporcionar la persona con DI que únicamente contar con la reportada por el cuidador principal, aunque lo ideal sería obtener información de ambas partes, como muy bien se plantea en el cuestionario LANTS 
(Wigham et al., 20I I). De este modo, aspectos que puedan pasar desapercibidos por la propia persona se pondrán de manifiesto a través de terceros y viceversa, pues el cuidador no siempre es sensible a los cambios emocionales de la persona con DI. Una vez más se hace evidente la necesidad de formación en los profesionales para discriminar este tipo de cambios (Rodríguez Herrero et al., 2013b).

Todo ello enlazaría con el tercer objetivo: determinar el tipo de intervenciones que se están empleando con las personas con DI que atraviesan un proceso de duelo. Conviene precisar que no todas las personas con DI que han sufrido la pérdida de alguien significativo necesitan una intervención especializada, ya que pueden desarrollar estrategias de afrontamiento saludables que les permitan afrontar el dolor como, por ejemplo, contar con información adecuada y precisa sobre la muerte, disponer en su entorno de alguien cercano (e.g., cuidador, familiar, profesional) como figura de referencia emocional y formativa y tener la oportunidad de participar en los rituales relacionados con la muerte (Read, 2005; Rodríguez Herrero et al., 2013c). De esta forma, de entre los cuatro tipos de estrategias propuestos por Read (2005) en su guía de acompañamiento (i.e., educación, participación, facilitación e intervenciones terapéuticas), la participación y facilitación pueden tener un papel central en el adecuado afrontamiento de este proceso.

Para aquellas situaciones en las que este tipo de apoyo no es suficiente, se han desarrollado diferentes intervenciones, principalmente a través de psicoterapia individual o terapia grupal; en ambos casos, los estudios no muestran una mayor efectividad de unas terapias sobre otras, pero sí apuntan a que el desarrollo de un espacio seguro para la persona en duelo -donde puede expresarse y adquirir conocimientos de un tema que por lo general es tabú- ayuda a romper la conspiración del silencio de la que hablábamos (ForresterJones, 20I3; Read y Elliott, 2007) y, por tanto, favorecen la inclusión de estas personas en el contexto vital en el que se desarrollan. Por otro lado, las intervenciones se centran cada vez más en el entorno más cercano de la persona (Campbell y Bell, 2010), pues se ha observado que trabajar inmerso en el contexto ayuda a disponer del apoyo necesario, el cual en numerosas ocasiones no se les proporciona por una falta de conocimiento por parte de los cuidadores. Se ha constatado que una vez han sido formados en el tema aumentan sus conocimientos, su confianza y su satisfacción laboral (Reynolds et al., 2008).

Por todo lo expuesto hasta el momento, podríamos afirmar que no contamos con un protocolo de actuación ante el duelo en personas con DI, aunque sí es cierto que tenemos ciertas guías (Galán, 20I4) que permiten ayudar al sujeto a sobrellevar el proceso de pérdida $\mathrm{y}$ a los profesionales a tener herramientas que favorezcan el apoyo a este colectivo y reduzcan el burnout que puede aparecer ante el desgaste emocional de estos trabajadores (Gilrane y Taggart, 2007; Gray y Abendroth, 20I6; Handley y Hutchinson, 20I3; Morgan y McEvoy, 20I4). Por tanto, se pone de manifiesto la necesidad, por un lado, de normalización de la muerte como un elemento educativo desde un enfoque previo-preventivo (Rodríguez Herrero et al., 2013 3 ) y, por otro, evaluar la eficacia de las intervenciones propuestas con el fin de garantizar el bienestar de la persona con DI y de su cuidador desde un enfoque paliativo, partiendo siempre de la necesidad de una intervención integral dentro del contexto natural.

En conclusión, aún son muchas las carencias de conocimiento que se presentan en el campo de la DI respecto al duelo. Es necesario trabajar en el desarrollo de investigaciones con muestras más amplias que permitan, además de un detallado conocimiento de las características del proceso en esta población, el desarrollo de una herramienta de uso profesional que ayude a determinar las necesidades de apoyo de las personas con DI que pasan por un proceso de duelo. Solo de este modo se podrá comenzar a trabajar de forma rigurosa hacia intervenciones y tratamientos eficaces, desarrollando prácticas basadas en evidencias. 


\section{Referencias bibliográficas}

Aguado. A. L. y Alcedo, M. A. (2004): "Necesidades percibidas en el proceso de envejecimiento de las personas con discapacidad intelectual". Psicothema, 35 (I): 5-I9.

American Psychiatric Association (2013): Diagnostic and Statistical Manual of Mental Disorders, Fifth Edition, Arlington, VA: American Psychiatric Association.

Barreto, P. et al. (201 2): "Detección de duelo complicado". Psicooncología, $9(2-3): 355-368$.

Bermejo, J. C. (2005): Estoy en duelo, Madrid: Editorial Promoción Popular Cristiana.

Blackman, N. (2008): “The development of an assessment tool for the bereavement needs of people with learning disabilities". British Journal of Learning Disabilities, 36 (3): I65I70.

Blackman, N. (2013). The Use of Psychotherapy in Supporting People with Intellectual Disabilities who Have Experienced Bereavement (tesis doctoral) (en línea). <https://uhra.herts.ac.uk/ bitstream/handle/2299/980I/07165752\%20 Blackman \% 20Noelle \% 20-\% 2ofinal \% 20 $\mathrm{PhD} \%$ 2osubmission.pdf? sequence $=\mathrm{I}>$, acceso I3 de diciembre de 2017.

Blackman, N. (20I6): "Supporting people with learning disabilities through a bereavement". Tizard Learning Disability Review, 2I: 199202.

Brickell, C. y Munir, K. (2008): "Grief and its complications in individual with intellectual disability". Harvard Review of Psychiatry, I6 (I): I-I 2 .

Bonell-Pascual, E. et al. (I999): "Bereavement and grief in adults with learning disabilities: a follow-up study". British Journal of Psychiatry, I75 (4): 348-350.

Borsay, C. et al. (2013): "Planning, facilitating and evaluating a bereavement group for adults with learning disabilities living in the community".
British Journal of Learning Disabilities, 4I (4): 266-272.

Boyden, P. et al. (2010): "Setting up and running a loss and bereavement support group for adults with learning disabilities". British Journal of Learning Disabilities, 38 (I): 35-40.

Brown, M. et al. (20I I): “A review of the literature relating to psychological interventions and people with intellectual disabilities: issues for research, policy, education and clinical practice". Journal of Intellectual Disabilities, I 5 (I): 3 I-45.

Campbell, A. y Bell, D. (20I0): “Sad, just sad: a woman with a learning disability experiencing bereavement". British Journal of Learning Disabilities, 39: I I-I6.

Cisternas, M. S. (20I 5): "Desafíos y avances en los derechos de las personas con discapacidad: una perspectiva global". Anuario de los Derechos Humanos, II: I7-37.

Clute, M. A. (20I0): "Bereavement interventions for adults with intellectual disabilities: what works?". Journal of Death and Dying, 6I (2): I63-I77.

Clute, M. A. y Kobayashi, R. (20I2): "Looking within and reaching out: bereavement counselor perceptions of grieving adults with ID”. American Journal of Hospice and Palliative Medicine, 29 (8): 583-590.

Díaz, P. et al. (20I4): Guía Duelo en Adultos, Madrid: Fundación Mario Losantos del Campo.

Dodd, P. C. (2007): Bereavement and people with intellectual disabilities (tesis doctoral). Dublín: Universidad de Dublin, Trinity College.

Dodd, P. C. et al. (2008): "A study of complicated grief symptoms in people with intellectual disabilities". Journal of Intellectual Disability Research, 52: 4I 5-425.

Dodd, P. C. y Guerin, S. (2009): “Grief and bereavement in people with intellectual 
disabilities". Current Opinion in Psychiatry, 22 (5): $442-446$.

Echeburúa, E. y Herrán, A. (2007): “¿Cuándo el duelo es patológico y cómo hay que tratarlo?”. Análisis y Modificación de Conducta, I 47 (33): 3I-5O.

Esbensen, A. J. et al. (2008): "Stability and change in health, functional abilities, and behavior problems among Adults with and without Down Syndrome". American Journal on Mental Retardation, I I 3: 263-277.

Fernández- Ríos, L. y Buela-Casal, G. (2009): "Standards for the preparation and writing of Psychology review articles". International Journal of Clinical and Health Psychology, 9 (2): 329-344.

Forrester-Jones, R. (2013): “The road barely taken: funerals, and people with intellectual disabilities". Journal of Applied Research in Intellectual Disabilities, 26 (3): 243-256.

Galán, J. C. (coord.) (20I4): Guía práctica de duelo para personas con discapacidad intelectual (en línea).

< http://www.atades.com/wp-content/ uploads/201 $5 / 06 / \mathrm{Gu} \% \mathrm{C}_{3} \% \mathrm{ADa}-$ pr\% $\mathrm{C}_{3} \%$ A I ctica-de-duelo-para-personas-comdiscapacidad-intelectual-FUNDAT.pdf $>$, acceso 3 de febrero de 2017.

García, J. (20 I2): “Manejo del duelo en atención primaria". Revista Digital de Medicina Psicosomática y Psicoterapia, 2: I-I8.

Garvía-Peñuelas, B. (2009): "Duelo y discapacidad". Revista Médica Internacional sobre el Sindrome de Down, I3 (I): I3-I6.

Gilrane, U. y Taggart, L. (2007): “An exploration of the support received by people with intellectual disabilities who have been bereaved". Journal of Research in Nursing, I 2: I29-I 44 .

Gray, J. A. y Abendroth, M. (2016): "Perspectives of US direct car workers on the grief process of persons with intellectual and developmental disabilities: implications for practice". Journal of Applied Research in Intellectual Disabilities, 29 (5), 468-480.
Gray, J. A. y Truesdale, J. (20I 5): “A delphi study on staff bereavement training in the intellectual and developmental disabilities field". Intellectual and Developmental Disabilities, 53 (6): 394-405.

Guerin, S. et al. (2009): “An initial assessment of the psychometric properties of the Complicated Grief Questionnaire for People with Intellectual Disabilities (CGQ-ID)". Research in Developmental Disabilities, 30: I 258-I 267.

Handley, E. y Hutchinson, N. (20I3): “The experience of carers in supporting people with intellectual disabilities through the process of bereavement: an interpretative phenomenological analysis". Journal of Applied Research in Intellectual Disabilities, 26 (3): I 86-I94.

Hernández, J. y Millán, J. M. (20I 5): “Las personas con discapacidad en España: inserción laboral y crisis económica". Revista Española de Discapacidad, 3 (I): 29-56.

Herrán, A. y Cortina, M. (2008a): “La educación para la muerte como ámbito formativo: más allá del duelo". Psicooncología, 5 (2-3): 409424 .

Herrán, A. y Cortina, M. (2008b): "La práctica del 'Acompañamiento Educativo' desde la tutoría en situaciones de duelo". Tendencias Pedagógicas, I3: I 57-I73.

Holey, J. N. y McKinney, C. (20I 5): “Music therapy in the bereavement of adults with intellectual disabilities: A clinical report". Music Therapy Perspectives, 33: 93-44.

Hollins, S. y Esterhuyzen, S. (I997): “Bereavement and grief in adults with learning disabilities". The British Journal of Psychiatry, I70 (6): 497$50 \mathrm{I}$.

Instituto Nacional de Estadística (2008): Encuesta sobre Discapacidades, Autonomía personal y situaciones de Dependencia 2008 (en línea). $<$ http://www.ine.es/dyngs/INEbase/es/operacion. htm?c=Estadistica_C\&cid $=\mathrm{I} 254736176782$ \&menu $=$ resultados\&idp $=$ I 254735573 I $75>$, acceso 20 de febrero de 2017.

LoConto, D. G. y Jones-Pruett, D. L. (2008): "Utilizing symbolic interactionism to assist 
people with mental retardation during the grieving process”. Illness, Crisis and Loss, I 6 (I): $2 \mathrm{I}-35$.

MacHale, R. et al. (2009): "Caregiver perceptions of the understanding of death and need for bereavement support in adults with intellectual disabilities". Journal of Applied Research in Intellectual Disabilities, 22: 574-58I.

Mappin, R. y Hanlon, D. (2005): “Description and evaluation of a bereavement group for people with learning disabilities". British Journal of Learning Disabilities, 33: I06-I I 2.

McEvoy, J. et al. (2OI2): "Concept of death and perceptions of bereavement in adults with intellectual disabilities". Journal of Intellectual Disability Research, 56 (2): I9I-203.

McEvoy, J. et al. (2017): “A matter of life and death: knowledge about the body and concept of death in adults with intellectual disabilities". Journal of Intellectual Disability Research, 6I: 89-98.

McRitchie, R. et al. (20I4): "How adults with an intellectual disability experience bereavement and grief: a qualitative exploration". Death Studies, 38: I79-I85.

Meza, E. G. et al. (2008): “El proceso del duelo. Un mecanismo humano para el manejo de las pérdidas emocionales". Revista de Especialidades Médico-Quirúrgicas, I3 (I): 28-3I.

Mevissen, L. et al. (2016): “Assessment and Treatment of PTSD in People with Intellectual Disabilities”, en Martin, C. R., Preedy, V. R. y Patel, V. B. (coords.): Comprehensive guide to post-traumatic stress disorders. Basel: Springer International Publishing.

Millán, J. C. (2002): Inclusión socio-laboral $y$ envejecimiento en las personas con discapacidad intelectual (en línea). <http:// gerontologia.udc.es/new/documents/ InclusionSocioLaboral.pdf >, acceso 28 de enero de 2017.

Morgan, N. y McEvoy, J. (20I4): "Exploring the bereavement experiences of older women with intellectual disabilities in long-term residential care: a staff perspective". Omega-Journal of

Death and Dying, 69 (2): I I 7-I35.

Muñoz, F. et al. (2002): "La familia en la enfermedad terminal (II)". Medicina de Familia, $3(4): 262-268$.

Neimeyer, R. A. (2002): Aprender de la pérdida. Una guía para afrontar el duelo, Barcelona: Paidós.

Organización Mundial de la Salud (200I): International Classification of Functioning, Disability and Health: ICF, Génova: Organización Mundial de la Salud.

Organización Mundial de la Salud (20I I): Informe mundial sobre la discapacidad (en línea).

<http://apps.who.int/iris/handle/I0665/75356>, acceso 5 de febrero de 2017.

Parkes, G. et al. (2007): "Referrals to an intellectual disability psychotherapy service in an inner city catchment area. A retrospective case notes study". Journal of Applied Research in Intellectual Disabilities, 20 (4):373-378.

Pereira, R. (2010): "Evolución y diagnóstico del duelo normal y patológico". Formación Médica Continuada de Atención Primaria, I7 (го), 656-663.

Read, S. (2005): “Loss, bereavement and learning disabilities: providing a continuum of support". Learning Disability Practice, 8 (I): 3 I-37.

Read, S. et al. (2013): "Using action research to design bereavement software: engaging people with intellectual disabilities for effective development". Journal of Applied Research in Intellectual Disabilities, 26 (3): I95-206.

Read, S. y Elliott, D. (2007): "Exploring continuum of support for bereaved people with intellectual disabilities: a strategic approach". Journal of Intellectual Disabilities, I I (2), I67-I 8I.

Reynolds, S. et al. (2008): "Evaluation of a bereavement training program for staff in an intellectual disabilities service". Journal of Policy and Practice in Intellectual Disabilities, 5: I-5.

Rodríguez Herrero, P. et al. (2013a): "Y si me muero... ¿dónde está mi futuro? Hacia una 
educación para la muerte en personas con discapacidad intelectual" (en línea).

$<$ http://revistas.uned.es/index.php/ educacionXXI/article/view/729>, acceso $6 \mathrm{de}$ febrero de 2017 .

Rodríguez Herrero, P. et al. (2013b): “Diseño, aplicación y evaluación de un programa de educación para la muerte dirigido a personas adultas con discapacidad intelectual". Revista Iberoamericana de Educación, 63: I99-2 I9.

Rodríguez Herrero, P. et al. (2013c): "Orientaciones pedagógicas para el acompañamiento educativo por duelo a personas adultas con discapacidad intelectual. Teoría de la Educación”. Revista Interuniversitaria, 25 (I): I73-I 89.

Rodríguez Herrero, P. et al. (20 I 5): “Concept of death in young people with intellectual disability: a contribution to the pedagogy on death". Siglo Cero, 46 (I): 67-80.

Ruiz, M. N. y Sainz, N. (2008): “Apoyo en el proceso de duelo de personas con discapacidad intelectual. Escuchar, ayudar y aconsejar es ser un amigo de verdad" (en línea).

< http://sid.usal.es/idocs/F8/FDO20846/nerea.pdf>, acceso Io de enero de 2017.

Schalock, R. L. et al. (2010): Intellectual disability. Definition, classification, and systems of supports. $I^{\text {th }}$ ed., Washington, D.C.: American Association on Intellectual and Development Disabilities.

Shear, M. K. (2OI 2): "Getting straight about grief". Depression and Anxiety, 29 (6): 46I-464.

Stancliffe, R. et al. (2016): "Knowing, planning for and fearing death: Do adults with intellectual disability and disability staff differ?”. Research in Developmental Disabilities, 49-50: 47-59.

Sttodart, K. P. et al. (2002): "Outcome evaluation of bereavement groups for adults with intellectual disabilities". Journal of Applied Research in Intellectual Disabilities, I 5 (I): 28-35.
Tizón, J. L. (2004): Pérdida, pena y duelo. Vivencias, investigación y asistencia, Barcelona: Paidós.

Uribe, S. (2015): "Comentarios al trastorno persistente de duelo complicado del DSM- 5 ". Revista Iberoamericana de Psicotraumatología y Disociación, 7 ( ( ).

Wakefield, J. (2013): “DSM-5 grief scorecard: Assessment and outcomes of proposals to pathologize grief". World Psychiatry, I 2 (2): I7I-I73.

Watters, L. et al. (2OI2): "The impact of staff training on the knowledge of support staff in relation to bereavement and people with an intellectual disability". British Journal of Learning Disabilities, 40 (3): 194-200.

Wiese, M. et al. (201 5 ): "Learning about dying, death, and end-of-life planning: current issues informing future actions". Journal of Intellectual and Developmental Disability, 40 (2): $230-235$.

Wigham, S. et al. (2OII): “The Lancaster and Northgate Trauma Scales (LANTS): the development and psychometric properties of a measure of trauma for people with mild to moderate intellectual disabilities". Research in Developmental Disabilities, 32 (6): 265 I-2659.

Young, H. (20I6): "Conceptualising bereavement in profund and multiple learning disabilities". Tizard Learning Disability Review, 2 I (4): I86I98.

Young, H. et al. (2016): "Making sense of bereavement in people with profound intellectual and multiple disabilities: carer perspectives". Journal of Applied Research in Intellectual Disabilities.

Young, H. y Garrard, B. (2015): "Bereavement and loss: developing a memory box to support a young woman with profound learning disabilities". British Journal of Learning Disabilities, 44: 78-84. 